\begin{tabular}{|c|l|}
\hline Title & Evidence for exchange interaction between donor and acceptor layers in $\beta$ '-(BEDT-TTF)(TCNQ) \\
\hline Author(s) & Eto, Y oshihiro; Kawamoto, A tsushi; Matsunaga, Noriaki; Nomura, Kazushige; Y amamoto, Kaoru; Y akushi, Ky uya \\
\hline Citation & $\begin{array}{l}\text { Physical Review B, 80(17), 174506 } \\
\text { https://doi.org/10.1103/PhysRevB.80.174506 }\end{array}$ \\
\hline Issue Date & 2009-11-01 \\
\hline Doc URL & http://hdl.handle.net/2115/42515 \\
\hline Rights & ○2009 The A merican Physical Society \\
\hline Type & article \\
\hline File Information & PRB80-17_174506.pdf \\
\hline
\end{tabular}

Instructions for use 


\title{
Evidence for exchange interaction between donor and acceptor layers in $\boldsymbol{\beta}^{\prime}$-(BEDT-TTF)(TCNQ)
}

\author{
Yoshihiro Eto, Atsushi Kawamoto, ${ }^{*}$ Noriaki Matsunaga, and Kazushige Nomura \\ Department of Quantum and Condensed Matter Physics, Graduate School of Science, Hokkaido University, Kita-ku, Sapporo, \\ Hokkaido 060-0810, Japan \\ Kaoru Yamamoto and Kyuya Yakushi \\ Institute for Molecular Science, Myodaiji, Okazaki, Aichi 444-8585, Japan
}

(Received 4 September 2009; published 6 November 2009)

\begin{abstract}
We assessed the infrared-absorption spectra and ${ }^{13} \mathrm{C}-\mathrm{NMR}$ measurements in a layered organic salt, $\beta^{\prime}$-(BEDT-TTF)(TCNQ), which exhibits antiferromagnetic transitions at 20 and $3 \mathrm{~K}$. The former originates from the spin in the bis-(ethylenedithio)-tetrathiafulvalene (BEDT-TTF) layers, while the latter originates from the localized spin in the tetracyanoquinodimethane (TCNQ) layers. Using infrared-absorption spectroscopy, we estimated the degree of charge transfer, $\rho$, between BEDT-TTF and TCNQ as 0.5 . Using ${ }^{13} \mathrm{C}-\mathrm{NMR}$ spectroscopy, we observed an exchange field at the BEDT-TTF site, which is produced by the localized spins of TCNQ dimers. Using the obtained value of $\rho$ and the molecular arrangement of $\beta^{\prime}$-(BEDT-TTF)(TCNQ), which is similar to that of the highest $T_{c}$ organic superconductor, $\beta^{\prime}$-(BEDT-TTF) ${ }_{2} \mathrm{ICl}_{2}$, we concluded that the absence of the pressure-induced superconductivity in $\beta^{\prime}$-(BEDT-TTF)(TCNQ) results from the presence of this exchange field. The exchange interaction, $J$, and the exchange field, $H_{e x}$, were estimated as $-12 \mathrm{~K}$ and $-19 \mathrm{~T} / \mu_{B}$ on the TCNQ dimer unit, respectively. These findings suggest that superconductivity may arise in $\beta^{\prime}$-(BEDT-TTF)(TCNQ) by the application of an external field of $19 \mathrm{~T}$ under high pressure.
\end{abstract}

DOI: $10.1103 /$ PhysRevB.80.174506

PACS number(s): 71.30.+h, 82.80.Gk, 76.60.-k

\section{INTRODUCTION}

Organic radical salts, (BEDT-TTF) 2 X, have been investigated for use as organic superconductors. For example, bis(ethylenedithio)-tetrathiafulvalene (BEDT-TTF), together with inorganic ions, form many conducting salts of various crystal structures. In terms of electrical conductivity, these salts range from superconductors to high-resistance semiconductors. They are classified by the arrangement of BEDTTTF molecules in the crystal and the type of structure and electronic properties are closely related. For example, $\beta$-type salts become superconductors, whereas $\beta^{\prime}$-type salts, which have strong dimeric structures, show semiconducting behavior, and $\beta^{\prime \prime}$-type salts show metallic conductivity down to low temperatures. In $\kappa$-type salts, the superconducting and antiferromagnetic (AF) insulating phases coexist at low temperatures. The $\beta^{\prime}$-type salt, $\beta^{\prime}$-(BEDT-TTF) ${ }_{2} \mathrm{ICl}_{2}$, shows antiferromagnetic transition at $22 \mathrm{~K}$ at ambient pressure. ${ }^{1} \mathrm{Al}-$ though it shows semiconducting behavior at ambient pressure, a superconducting transition occurs at $14.2 \mathrm{~K}$ under $8.2 \mathrm{GPa}^{2}$ This is the highest known transition temperature among organic superconductors. Fluctuation of antiferromagnetism has also been proposed as the mechanism of superconductivity in this salt. ${ }^{3,4}$

A second $\beta^{\prime}$-type salt, $\beta^{\prime}$-(BEDT-TTF)(TCNQ) is thought to be similar to $\beta^{\prime}$-(BEDT-TTF) ${ }_{2} \mathrm{ICl}_{2}$, because their molecular arrangements are the same (Fig. 1). In addition, each of BEDT-TTF and tetracyanoquinodimethane (TCNQ) molecules forms a dimeric structure, with the TCNQ molecules forming a one-dimensional stack. ${ }^{5}$ The difference between $\beta^{\prime}$-(BEDT-TTF)(TCNQ) and $\beta^{\prime}$-(BEDT-TTF) ${ }_{2} \mathrm{ICl}_{2}$ is in the counter anion, which is organic in $\beta^{\prime}$-(BEDT-TTF)(TCNQ) but inorganic in $\beta^{\prime}$-(BEDT-TTF $)_{2} \mathrm{ICl}_{2}$. Spin-susceptibility and NMR measurements have revealed that, under ambient pressure, $\beta^{\prime}$-(BEDT-TTF)(TCNQ) experiences an antiferro- magnetic transition at $20 \mathrm{~K}$ at the BEDT-TTF site and a second transition at $3 \mathrm{~K}$ at the TCNQ site. ${ }^{6-8}$ However, these ${ }^{13} \mathrm{C}$-NMR measurements were performed with powdered samples, so the properties of the antiferromagnetic phase of this salt are unclear. ${ }^{7} \quad \beta^{\prime}$-(BEDT-TTF)(TCNQ) and $\beta^{\prime}$-(BEDT-TTF) ${ }_{2} \mathrm{ICl}_{2}$ have the same $\beta^{\prime}$-type molecular arrangement and show antiferromagnetic ordering at their BEDT-TTF sites at nearly the same temperature. At ambient pressure, the electrical conductivity of $\beta^{\prime}$-(BEDT-TTF)(TCNQ) shows a metal-insulator (MI) transition at $330 \mathrm{~K}$, and the transition temperature decreases by applying pressure up to about $1.1 \mathrm{GPa} .{ }^{8}$ Although superconducting transition in the $\mathrm{ICl}_{2}$ salt occurs at $14.2 \mathrm{~K}$ at $8.2 \mathrm{GPa}$, the superconductivity in the TCNQ salt under high pressure has not been determined. We, therefore, assessed the
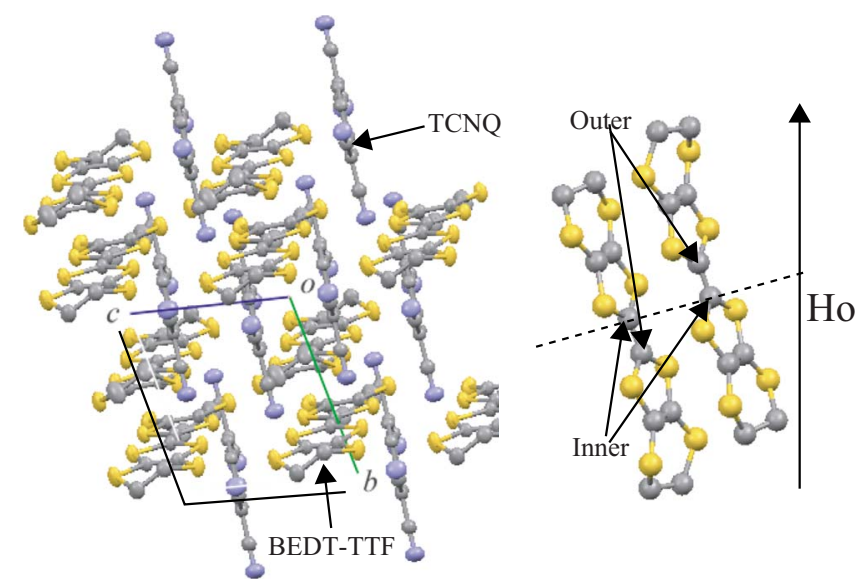

FIG. 1. (Color online) Left: crystal structure of $\beta^{\prime}$-(BEDT-TTF)(TCNQ) with projection along $a$. Right: dimeric structure with site definition of central $\mathrm{C}=\mathrm{C}$ carbons. 
infrared-absorption spectra and ${ }^{13} \mathrm{C}-\mathrm{NMR}$ measurements of $\beta^{\prime}$-(BEDT-TTF)(TCNQ) to determine its electronic structure and the appearance of superconductivity.

\section{EXPERIMENT}

We utilized a single crystal of $\beta^{\prime}$-(BEDT-TTF)(TCNQ), in which one side of the central carbon atoms in the BEDTTTF molecules were replaced by ${ }^{13} \mathrm{C}$ nuclei. ${ }^{9} \mathrm{By}$ using this molecule, we could prevent the splitting caused by Pake doublets, while easily performing quantitative experiments. The crystal was prepared by the diffusion method with BEDTTTF and TCNQ molecules in 1,1,2-trichrolo-ethane. We confirmed its $\beta^{\prime}$ structure and determined its crystal orientation by x-ray diffraction. Room-temperature (RT) optical conductivity was achieved by the Kramers-Kronig transformation of the E $\| a$ reflectivity using the nonenriched BEDT-TTF molecule. The $a$ axis is perpendicular to the conducting layer. NMR measurements were performed with decreasing temperature under a field of $9.4 \mathrm{~T}$ and under pressures ranging from ambient pressure to $0.8 \mathrm{GPa}$ using a clamp cell made of $\mathrm{BeCu}$ alloy. Daphne oil 7373 was used as a pressure medium. NMR spectra were obtained by fast Fourier transformation of the echo signal with a $\pi / 2-\pi$ pulse sequence. The pulse width of $\pi / 2$ is typically $4 \mu$ s. Spin-lattice-relaxation time was determined by the saturation recovery method. The crystal was characterized by in-plane electrical conductivity measurements under pressures up to $1.0 \mathrm{GPa}$ using the conventional four-terminal method.

\section{RESULTS AND DISCUSSION}

\section{A. Degree of charge transfer between BEDT-TTF and TCNQ molecules}

In $\beta^{\prime}$-(BEDT-TTF) ${ }_{2} \mathrm{ICl}_{2}, \mathrm{ICl}_{2}^{-}$is a monovalent anion, making the formal charge on each BEDT-TTF molecule +0.5 . In contrast, $\beta^{\prime}$-(BEDT-TTF)(TCNQ) is a chargetransfer complex of BEDT-TTF and TCNQ, so it has a freedom of charge transfer, $\rho$. We, therefore, hypothesized that $\beta^{\prime}$-(BEDT-TTF)(TCNQ) will show no superconductivity because the formal charge on BEDT-TTF molecules is different from that on $\mathrm{ICl}_{2}$ molecules. As a result, the two salts will vary with respect to band filling and shifting in Fermi surfaces. To determine the degree of charge transfer of $\beta^{\prime}$-(BEDT-TTF)(TCNQ), we measured its infraredabsorption spectrum. We estimated the degree of charge transfer using the $\nu_{19}$ mode, which contributes to the stretching of the $\mathrm{C} \equiv \mathrm{N}$ bond in the TCNQ molecule. The frequency of the $\nu_{19}$ mode depends on the electrons in the lowest unoccupied molecular orbital (LUMO). Previous studies have shown that the frequency of $\nu_{19}$ on neutral molecules is $2228 \mathrm{~cm}^{-1}$ and that on the ionic molecules is $2181 \mathrm{~cm}^{-1} .{ }^{10}$ With a formal charge of $-0.5, \nu_{19}$ is expected to be midway between these values, or $2205 \mathrm{~cm}^{-1}$. Thus, the peak position of $\nu_{19}$ will correspond to the degree of charge transfer on the TCNQ molecule.

Figure 2(a) shows the infrared-absorption spectrum of $\beta^{\prime}$-(BEDT-TTF)(TCNQ) at room temperature. $\nu_{19}$ is observed at $2202 \mathrm{~cm}^{-1}$. Since this is almost the same as the
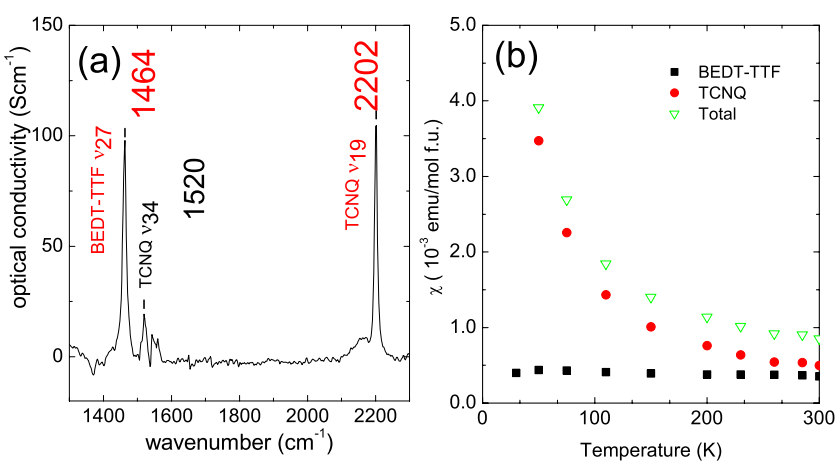

FIG. 2. (Color online) (a) RT optical conductivity obtained from the Kramers-Kronig transformation of the E $\| a$ reflectivity. The $a$ axis is perpendicular to the conducting layer. (b) Magneticsusceptibility decomposition into BEDT-TTF and TCNQ sites (Ref. 8).

result from a formal charge of -0.5 , the degree of formal charge on the TCNQ site is -0.5 . In a similar way, we estimated the degree of formal charge on the BEDT-TTF site as +0.5 from its vibration mode, $\nu_{27}$, of $1464 \mathrm{~cm}^{-1} .{ }^{11}$ In addition, the frequency of the corresponding mode did not show a strong dependence on temperature within $5 \mathrm{~cm}^{-1}$. These results indicate that the degree of charge transfer $\rho$ is 0.5 and is temperature independent. In a typical charge-transfer complex, for example, TTF-TCNQ, due to the segregated column structure, two types of band from the highest occupied molecular orbital (HOMO) of TTF and LUMO of TCNQ exist. To balance the chemical potential, partial charge transfer between HOMO of TTF and LUMO of TCNQ takes place [Fig. 3(a)]. In contrast, from the Curie-Weiss behavior of the spin susceptibility of the TCNQ site shown in Fig. 2(b), ${ }^{8}$ the temperature independence of $\left(T_{1}\right)^{-1}$ (Refs. 6 and 7) and its formal charge of -0.5 , the TCNQ site is expected to be a dimerMott insulator, in which one electron is localized to a dimer. Rather the LUMO band of TCNQ splits into lower and upper Hubbard bands and a Fermi level is present only on the BEDT-TTF band [Fig. 3(b)]. Therefore, the formal charge on the BEDT-TTF site is +0.5 and the BEDT-TTF layer seems to have the same electronic structure as the $\mathrm{ICl}_{2}$ salt. An alternative explanation is, therefore, needed for the differences between the $\mathrm{ICl}_{2}$ and TCNQ salts.

\section{B. NMR under pressure and phase diagram}

To investigate the electronic structure of $\beta^{\prime}$-(BEDT-TTF)(TCNQ), we performed ${ }^{13} \mathrm{C}$-NMR measure-

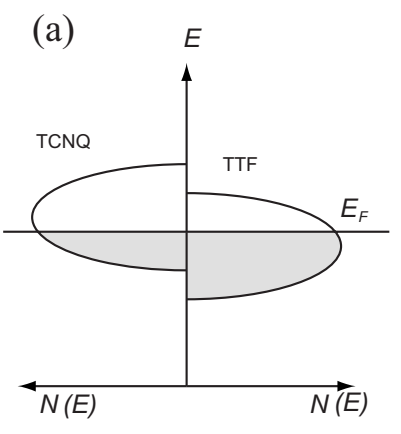

FIG. 3. Energy structure $\beta^{\prime}$-(BEDT-TTF)(TCNQ).

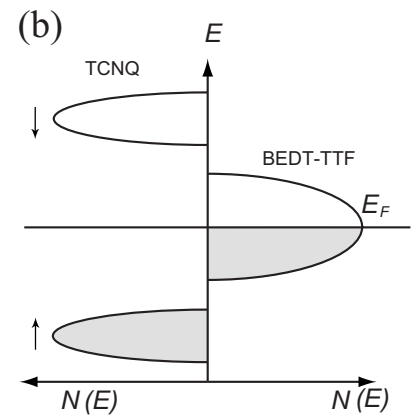

of (a) TTF-TCNQ and (b) 

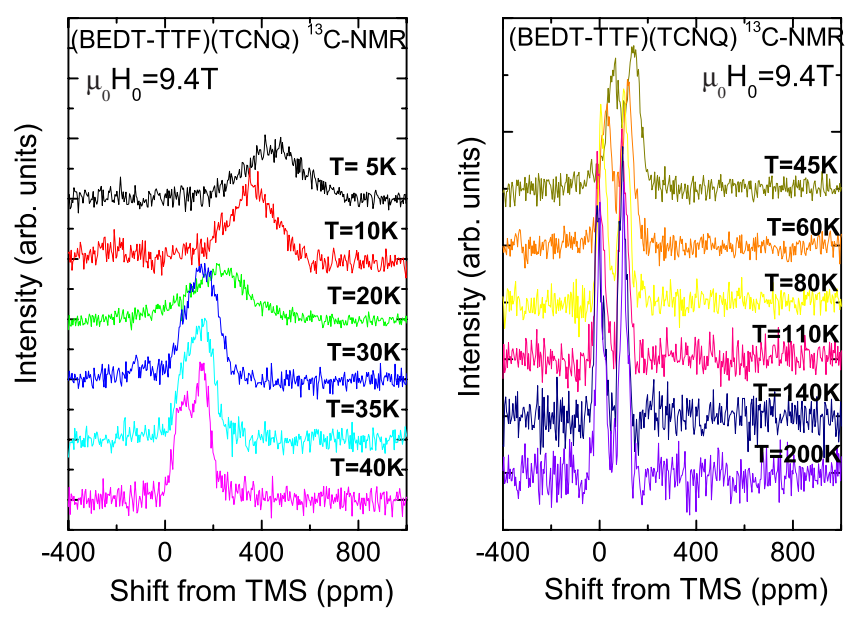

FIG. 4. (Color online) NMR spectrum of $\beta^{\prime}$-(BEDT-TTF)(TCNQ) at ambient pressure.

ments on the BEDT-TTF layer at ambient pressure and 0.3, 0.5 , and $0.8 \mathrm{GPa}$. Figure 4 shows the temperature dependence of the spectrum at ambient pressure. As BEDT-TTF forms a dimer in the sheet, there are two crystallographic nonequivalent inner and outer sites, as shown in Fig. 1. Therefore, we expected to observe two peaks, one each at the inner and outer sites. Indeed, as shown in Fig. 4, two peaks were observed in the high-temperature range. With decreasing temperature, the linewidths of the peaks increased, and one peak was observed at the antiferromagnetic transition temperature, $T_{N}$, of $20 \mathrm{~K}$. As the temperature was further decreased, the linewidths of the peaks increased. If the antiferromagnetic structure is commensurate, splitting of the spectrum below $T_{N}$ was expected, due to the difference in the internal magnetic field. We searched the spectrum in the range of $\pm 1 \mathrm{MHz}$ from the central frequency (100.703 $\mathrm{MHz})$ at $10 \mathrm{~K}$. However, only one peak was observed. The intensity ratio of spectrum $(I) \times$ temperature $(T)$ before and after transition, or $(I \times T)_{T=10 \mathrm{~K}} /(I \times T)_{T=23 \mathrm{~K}}$, corresponding to the number of spins, was $\approx 0.9$. It is, therefore, unlikely that there are other peaks outside this spectrum range. We performed the same measurements applying an external field parallel to the conduction layer and observed the same results in the perpendicular direction. Moreover, the linewidth of the peak increased after the transition. These results strongly suggest that the antiferromagnetic structure of $\beta^{\prime}$-(BEDT-TTF)(TCNQ) is incommensurate with spindensity wave (SDW). A SDW was premised on the theory that considers the dimensionality of the band. ${ }^{3,4}$ As in many organic conductors, the flopping field is less than $10 \mathrm{kOe}$. Thus, the moment is thought to be perpendicular to the external magnetic field of $9.4 \mathrm{~T}$. Therefore, the internal magnetic field is made via the off-diagonal term of the hyperfine coupling tensor. Using the value of the $\mathrm{ICl}_{2}$ salt, 0.1 $\sim 0.6 \mathrm{kOe} / \mu_{B}$ on the BEDT-TTF dimer, the amplitude of the moment of SDW was estimated as $0.04 \sim 0.2 \mu_{B}$ per molecule.

Results similar to those at ambient pressure were observed at both 0.5 and $0.8 \mathrm{GPa}$. That is, two peaks were observed in the high-temperature range; with decreasing temperature, the linewidths of the peaks increased and the
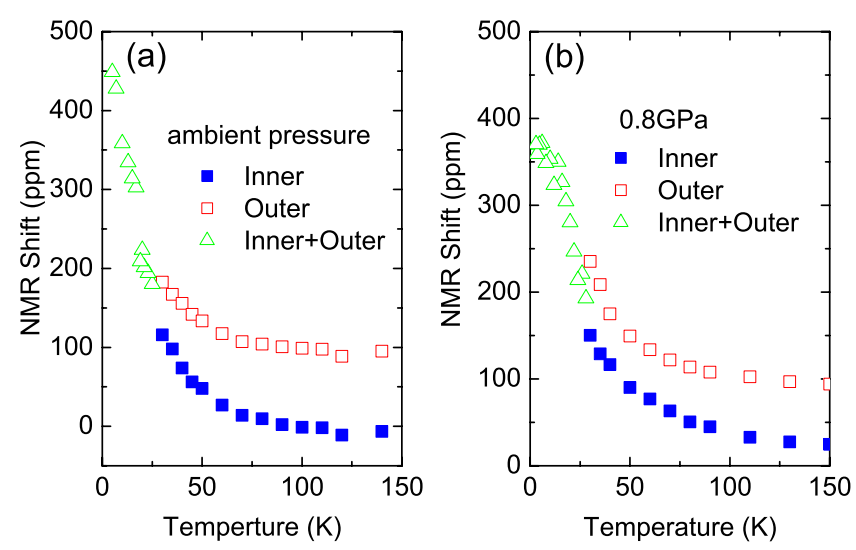

FIG. 5. (Color online) Temperature dependence of the NMR shift at (a) ambient pressure and (b) 0.8GPa.

two peaks overlapped; and, as the temperature further decreased, the linewidths of the peaks increased. Figure 5 shows the NMR shift in two peaks from tetra methyl silane. The temperature dependence of the NMR shift below $50 \mathrm{~K}$ was almost independent of pressure. As the overall features were the same as under ambient pressure, the antiferromagnetic structure was also expected to be incommensurate under pressure.

Since $T_{N}$ could not be determined from the spectrum where the two peaks merged at low temperature, the pressure dependence of $T_{N}$ was determined as the temperature at which $\left(T_{1} T\right)^{-1}$ diverged. Figure 6(a) shows the temperature dependence of $\left(T_{1} T\right)^{-1}$ at ambient pressure and under pressure. Divergence of $\left(T_{1} T\right)^{-1}$ was observed to be $23 \mathrm{~K}$ at 0.3 $\mathrm{GPa}$ and at $26 \mathrm{~K}$ at $0.5 \mathrm{GPa}$, which decreased to $3 \mathrm{~K}$ at 0.8 GPa. $T_{N}$ increased at pressures up to $0.5 \mathrm{GPa}$ but decreased rapidly at pressures above $0.5 \mathrm{GPa}$, indicating that $T_{N}$ is suppressed at high pressure. Such behavior of $T_{N}$ is predicted by the theoretical calculation that takes into account the change in dimensionality of the band structure. ${ }^{3,4}$ In Fig. 6(b), our results are plotted onto a $\mathrm{P}-\mathrm{T}$ phase diagram of electrical conductivity measurements from two groups. ${ }^{8,12}$ Although Iwasa et $_{\text {al. }}{ }^{8}$ suggested that $T_{N}$ was thought to connect the metal-insulator transition line at high pressure, our
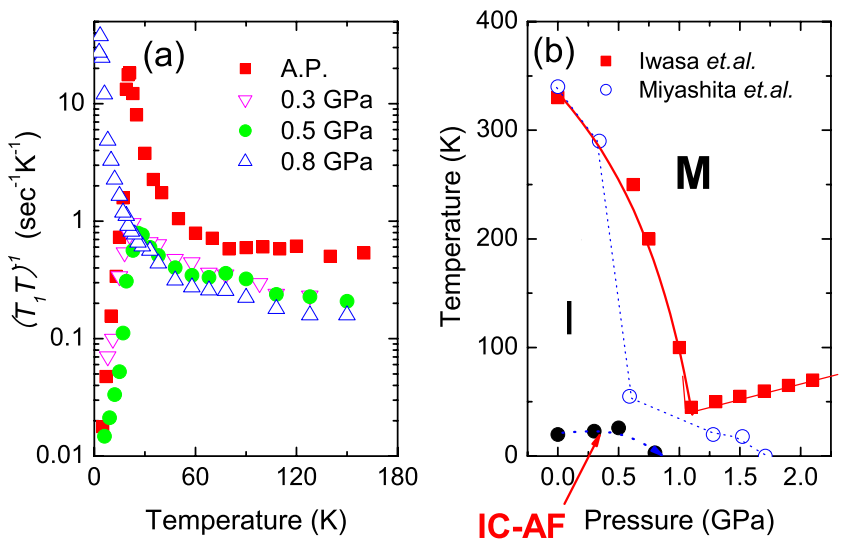

FIG. 6. (Color online) (a) Temperature dependence of $\left(T_{1} T\right)^{-1}$ of $\beta^{\prime}$-(BEDT-TTF)(TCNQ) at ambient pressure and under pressure (b) $\mathrm{P}-\mathrm{T}$ phase diagram of $\beta^{\prime}$-(BEDT-TTF)(TCNQ). 


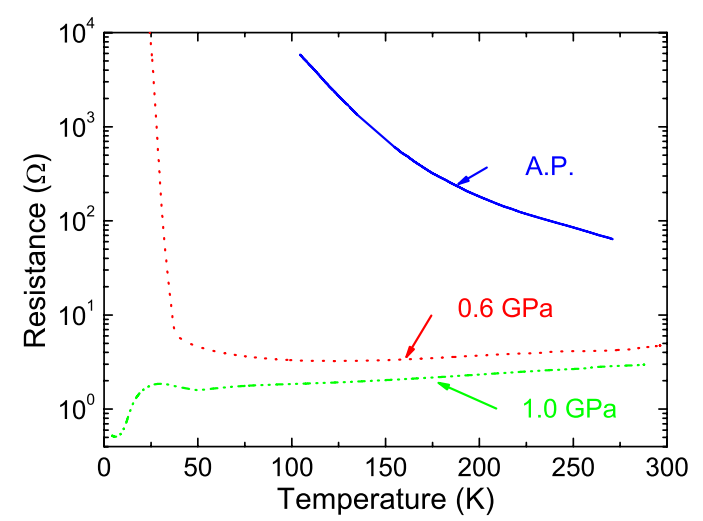

FIG. 7. (Color online) Temperature dependence of the resistance of $\beta^{\prime}$-(BEDT-TTF)(TCNQ) at ambient pressure and high pressure.

results suggest that there is no connection between the metalinsulator transition line and $T_{N}$ above $1 \mathrm{GPa}$. On the other hand, Miyashita et al. ${ }^{12}$ claimed that metallic behavior has been observed at low temperatures and at 1.0 GPa. Considering that the MI transition line from electrical conductivity is not connected with the line of the AF transition, MI transition at $50 \sim 100 \mathrm{~K}$ above $1.0 \mathrm{GPa}$ is unlikely. We confirmed that the electrical conductivity under pressures showed the same behavior as in Miyashita et al. (Fig. 7). Metallic behavior was clearly observed at low temperatures and at $1 \mathrm{GPa}$.

\section{Exchange field on BEDT-TTF from the magnetism of TCNQ}

Although the theoretical calculation suggested that superconductivity occurs in the region at which the antiferromagnetic transition is suppressed, superconductivity has not been observed in this salt. To explain why this salt does not show superconductivity under pressure, we focused on the NMR shift: NMR shift $\delta$ is generally expressed by the equation

$$
\delta=K+\sigma=A \chi_{E T}+\sigma,
$$

where $K$ is the Knight shift, $\sigma$ is the chemical shift, $A$ is the hyperfine coupling constant of the BEDT-TTF site, and $\chi_{E T}$ is the local spin susceptibility of the BEDT-TTF site. As shown in Fig. 2(b), electron spin-resonance studies have indicated that the spin susceptibility of the BEDT-TTF site is almost constant. ${ }^{8}$ In contrast, we found that the NMR shift increased rapidly below $50 \mathrm{~K}$ under all pressures. The NMR shift in the normal and antiferromagnetic phases seem to show Curie-type behavior, as does the spin susceptibility of the TCNQ site. This difference can be explained by assuming an exchange field from the TCNQ site, which is not present in the $\mathrm{ICl}_{2}$ salt. Including the contribution from the magnetism of the TCNQ site, the local field of the ${ }^{13} \mathrm{C}$ site can be described by the equation

$$
H_{\text {local }}=\sigma H_{0}+K\left(H_{0}+H_{e x}^{\mathrm{TCNQ}}\right)+H_{d i p}^{\mathrm{TCNQ}},
$$

where $H_{0}, H_{e x}^{\mathrm{TCNQ}}$, and $H_{d i p}^{\mathrm{TCNQ}}$ indicate the external, exchange, and dipolar fields, respectively, from the TCNQ site.

Since the ${ }^{13} \mathrm{C}$ site is on the central position on the BEDTTTF molecule and since TCNQ is a bulky anion, any ob-
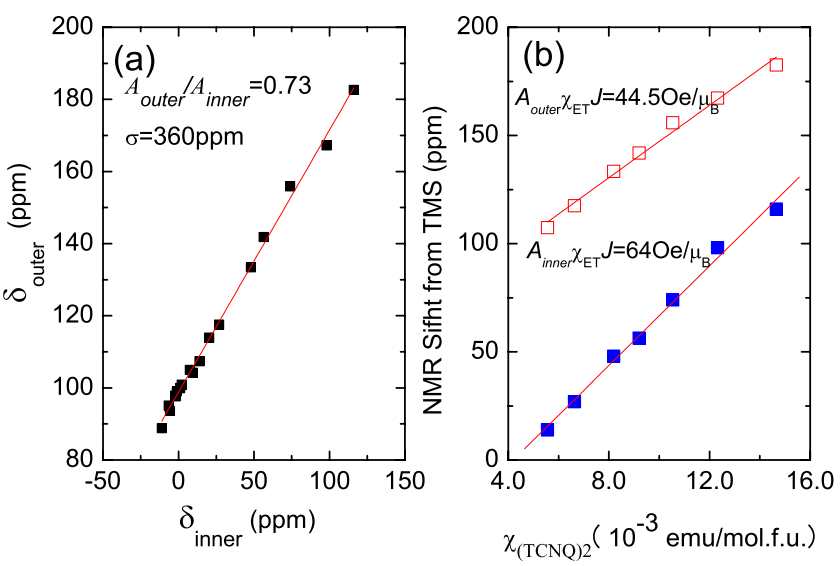

FIG. 8. (Color online) $\beta^{\prime}$-(BEDT-TTF)(TCNQ) $\beta^{\prime}$-(BEDT-TTF)(TCNQ).

(b)

$\begin{array}{ccc}\delta_{\text {inner }}-\delta_{\text {outer }} & \text { plot } & \text { of } \\ \delta-\chi_{(\mathrm{TCNQ})_{2}} & \text { plot } & \text { of }\end{array}$

served dipolar field is expected to be small. We estimated $H_{d i p}^{\mathrm{TCNQ}} / M_{\mathrm{TCNQ}}=-20 \sim 4 \mathrm{Oe} / \mu_{B}$, which includes the demagnetization field from the planiform crystal shape, ${ }^{13}$ here $M_{\mathrm{TCNQ}}$ is the magnetization on a TCNQ dimer. The uncertainty is due to the ambiguity of the demagnetization factor and this term can be ignored. Therefore, the above equation is simplified to

$$
\begin{aligned}
& H_{\text {local }} \approx \sigma H_{0}+K\left(H_{0}+H_{e x}\right) \\
& \quad=A \chi_{E T} H_{0}+\sigma H_{0}+A \chi_{E T} J \chi_{\mathrm{TCNQ}} H_{0},
\end{aligned}
$$

where $J$ is the spin coupling constant and $\chi_{\mathrm{TCNQ}}$ is the spin susceptibility of the TCNQ site. By dividing both sides of the equation by $H_{0}$, NMR shift can be represented as

$$
\delta=A \chi_{E T}+\sigma+A \chi_{E T} J \chi_{\mathrm{TCNQ}} .
$$

The first term is the Knight shift, the second term is the chemical shift, and the third term is the additional shift from the exchange interaction. We can estimate $\sigma$ from the above equation. Using the NMR shifts in the inner and outer sides, $\delta_{\text {inner }}$ and $\delta_{\text {outer }}$, we obtain the equation

$$
\delta_{\text {outer }}=\frac{A_{\text {outer }}}{A_{\text {inner }}} \delta_{\text {inner }}+\sigma\left(1-\frac{A_{\text {outer }}}{A_{\text {inner }}}\right) .
$$

As shown in Fig. 8(a), from the slope and intercept of the $\delta_{\text {inner }}-\delta_{\text {outer }}$ plot, we found that $\frac{A_{\text {outer }}}{A_{\text {inner }}}$ was $0.73(1)$ and $\sigma$ was $360(12) \mathrm{ppm}$. As mentioned above, $\chi_{E T}$ is almost temperature independent, such that the change in shift below $40 \mathrm{~K}$ is primarily due to $\chi_{\mathrm{TCNQ}}$. As shown in Fig. $8(\mathrm{~b})$, from the slope of the $\delta$ - $\chi_{\mathrm{TCNQ}}$ plot, we can estimate $A_{\text {inner }} \chi_{E T} J$ and $A_{\text {outer }} \chi_{E T} J$ as 64(2) Oe/ $\mu_{B}$ and 45(2) Oe/ $\mu_{B}$, respectively, on the TCNQ dimer unit. Since the magnetization of TCNQ is small in the high-temperature range ${ }^{8}$ the third term of the above equation can be ignored at room temperature.

By subtracting the value of the chemical shift, $360 \mathrm{ppm}$, from the NMR shifts, we calculated $A_{\text {inner }} \chi_{E T}$ and $A_{\text {outer }} \chi_{E T}$ as $-340 \mathrm{ppm}$ and $-250 \mathrm{ppm}$, respectively. To estimate $J$, we must consider the contribution from $H_{d i p}^{\mathrm{TCNQ}}$, which depends on the crystal shape. We can evaluate $H_{d i p}^{\mathrm{PCNQ}} / M_{\mathrm{TCNQ}}$ experimentally. From the equations that included $H_{d i p}^{\mathrm{TCNQ}}$, 
$A_{\text {outer }} \chi_{E T} J+H_{\text {dip }}^{\mathrm{TCNQ}} / M_{\mathrm{TCNQ}}=45 \mathrm{Oe} / \mu_{B}$ and $A_{\text {inner }} \chi_{E T} J$ $+H_{\text {dip }}^{\mathrm{TCNQ}} / M_{\mathrm{TCNQ}}=64 \mathrm{Oe} / \mu_{B}$ and the values of $A_{\text {inner }} \chi_{E T}$ and $A_{\text {outer }} \chi_{E T}, J$ and $H_{\text {dip }}^{\mathrm{TCNQ}} / M_{\mathrm{TCNQ}}$ were estimated to be $-12.2 \mathrm{~K}$ and $2.6 \mathrm{Oe} / \mu_{B}$, respectively. The value of $H_{d i p}^{\mathrm{TCNQ}} / M_{\mathrm{TCNQ}}$ is consistent with our rough estimations and is very small. A negative value of $J$ indicates that the exchange field is in the direction opposite to the external field. This exchange field acts to suppress the emergence of superconductivity and the metallic state under pressure. The negative $J$ suggests that the field induces superconductivity under pressure by the same mechanism as $\lambda$-(BETS $)_{2} \mathrm{FeCl}_{4}$ and $\kappa$-(BETS $)_{2} \mathrm{FeBr}_{4}$, the so-called Jaccarino-Peter effect. ${ }^{14-16}$ The exchange field at low temperature was estimated to be about $-19 \mathrm{~T}$. Organic superconductors show large $\mathrm{H}_{c 2}$ in the field parallel to the conducting plane. If the $\mathrm{H}_{c 2}$ exceeds 19 $\mathrm{T}$, the superconductivity may be observed by applying an external field of about $19 \mathrm{~T}$ under pressure. Compared to a $J$ value of $-2.3 \mathrm{~T} / \mu_{B}$ observed for $\kappa$-(BETS) ${ }_{2} \mathrm{FeBr}_{4}$ (Ref. 17) and $-6.4 \mathrm{~T} / \mu_{B}$ observed for $\lambda$-(BETS $)_{2} \mathrm{FeCl}_{4},{ }^{13}$ the $J$ value of $-19 \mathrm{~T} / \mu_{B}$ observed for $\beta^{\prime}$-(BEDT-TTF)(TCNQ) is larger. The difference may be due to the prevention of exchange coupling by $\mathrm{Cl}$ or $\mathrm{Br}$ atoms around $\mathrm{Fe}$ spins in $\lambda$-(BETS $)_{2} \mathrm{FeCl}_{4}$ or $\kappa$-(BETS $)_{2} \mathrm{FeBr}_{4}$, making an exchange field on the BETS molecule. In $\beta^{\prime}$-(BEDT-TTF)(TCNQ), however, the interaction is enhanced because BEDT-TTF molecules are next to TCNQ molecules. Large $J$ values in charge-transfer complexes may lead to the development of organic conductors with a new category of electronic properties.

\section{SUMMARY}

In summary, using infrared spectrum measurements, we estimated the degree of charge transfer between BEDT-TTF and TCNQ sites as 0.5, suggesting that the BEDT-TTF layer has the same electronic structure as the $\mathrm{ICl}_{2}$ salt. From ${ }^{13} \mathrm{C}-\mathrm{NMR}$ measurements, we found that $T_{N}$ increases at pressures up to $0.5 \mathrm{GPa}$ but decreases rapidly at higher pressures, up to $0.8 \mathrm{GPa}$, suggesting that antiferromagnetic transition is suppressed at high pressure. The existence of the exchange field on the BEDT-TTF site, produced by the spin of TCNQ sites can be expected from the temperature dependence of the NMR shift, and this exchange field is likely to suppress the emergence of superconductivity under pressure. Thus, unlike the $\mathrm{ICl}_{2}$ salt, the (BEDT-TTF)(TCNQ) salt does not become a superconductor simply by applying pressure. We estimated $J$ to be about $-12 \mathrm{~K}$ and the exchange field as $19 \mathrm{~T} / \mu_{B}$. The negative $J$ suggested that superconductivity may emerge by applying an external field of about $19 \mathrm{~T}$ under pressure. Future studies are required to validate the field-induced superconductivity under pressure.

\section{ACKNOWLEDGMENTS}

The authors thank K. Kumagai and Y. Furukawa of Hokkaido University, H. Taniguchi of Saitama University, and K. Hiraki of Gakusyuin University for stimulating discussions. This study was supported in part by a Grant-in-Aid for Scientific Research (Grant No.18540306) from the Ministry of Education, Culture, Sports, Science, and Technology, Japan.

\footnotetext{
*atkawa@phys.sci.hokudai.ac.jp

${ }^{1}$ N. Yoneyama, A. Miyazaki, T. Enoki, and G. Saito, Synth. Met. 86, 2029 (1997).

${ }^{2}$ H. Taniguchi, M. Miyashita, K. Uchiyama, K. Satoh, N. Mori, H. Okamoto, K. Miyagawa, K. Kanoda, M. Hedo, and Y. Uwatoko, J. Phys. Soc. Jpn. 72, 468 (2003).

${ }^{3}$ H. Kontani, Phys. Rev. B 67, 180503(R) (2003).

${ }^{4}$ T. Miyazaki and H. Kino, Phys. Rev. B 68, 220511(R) (2003).

${ }^{5}$ T. Mori and H. Inokuchi, Solid State Commun. 59, 355 (1986).

${ }^{6}$ K. Ishida, Y. Niino, Y. Kitaoka, K. Asayama, Y. Iwasa, and Y. Tokura, Synth. Met. 70, 881 (1995).

${ }^{7}$ A. Kawamoto, K. Miyagawa, A. Shimizu, and K. Kanoda, Synth. Met. 85, 1601 (1997).

${ }^{8}$ Y. Iwasa, K. Mizuhashi, T. Koda, Y. Tokura, and G. Saito, Phys. Rev. B 49, 3580 (1994).

${ }^{9}$ M. Yamashita, A. Kawamoto, and K. Kumagai, Synth. Met. 133-134, 125 (2003).

${ }^{10}$ R. Bozio, A. Girlando, and C. Pecile, J. Chem. Soc., Faraday
}

Trans. 2 71, 1237 (1975).

${ }^{11}$ T. Yamamoto, M. Uruichi, K. Yamamoto, K. Yakushi, A. Kawamoto, and H. Taniguchi, J. Phys. Chem. B 109, 15226 (2005).

${ }^{12}$ M. Miyashita, K. Uchiyama, H. Taniguchi, K. Satoh, Y. Uwatoko, N. Tajima, M. Tamura, and R. Kato, J. Phys. IV 114, 333 (2004).

${ }^{13}$ K. Hiraki, H. Mayaffre, M. Horvatic, C. Berthier, S. Uji, T. Yamaguchi, H. Tanaka, A. Kobayashi, H. Kobayashi, and T. Takahashi, J. Phys. Soc. Jpn. 76, 124708 (2007).

${ }^{14}$ V. Jaccarino and M. Peter, Phys. Rev. Lett. 9, 290 (1962).

${ }^{15}$ S. Uji, H. Shinagawa, T. Terashima, T. Yakabe, Y. Terai, M. Tokumoto, A. Kobayashi, H. Tanaka, and H. Kobayashi, Nature (London) 410, 908 (2001).

${ }^{16}$ H. Fujiwara, H. Kobayashi, E. Fujiwara, and A. Kobayashi, J. Am. Chem. Soc. 124, 6816 (2002).

${ }^{17}$ S. Fujiyama, M. Takigawa, J. Kikuchi, H.-B. Cui, H. Fujiwara, and H. Kobayashi, Phys. Rev. Lett. 96, 217001 (2006). 